\title{
Electrodynamic Calculations of the 4-Rod-RFQ Resonator for the Heidelberg High Current Injector
}

\author{
H. Podlech, National Superconducting Cyclotron Laboratory, East Lansing, MI-48824, USA \\ M. Grieser, R.v. Hahn, R. Repnow, D. Schwalm \\ Max-Planck-Institut für Kernphysik, 69117 Heidelberg, Germany
}

\begin{abstract}
At the Max-Planck-Institut für Kernphysik, a new type of accelerator is under operation which consists of a CHORDIS and an ECR ion source, two directly coupled 4-Rod-RFQ and eight 7-gap resonators [1]. The RFQs are designed to accelerate ions with a minimum charge to mass ratio of $\mathrm{q} / \mathrm{A}=1: 9$ up to an energy of $0.478 \mathrm{MeV} / \mathrm{u}$. Both RFQ resonators operate at a frequency of $108.48 \mathrm{MHz}$ with a maximum pulsed power of $90 \mathrm{~kW}$ (25\% duty factor).

The development of the RFQ resonators was accompanied by extensive numerical simulations with the code MAFIA [2]. We are presenting the results of the electrostatic calculations with modulated electrodes and the results of the electrodynamic simulations of the RFQ resonators.
\end{abstract}

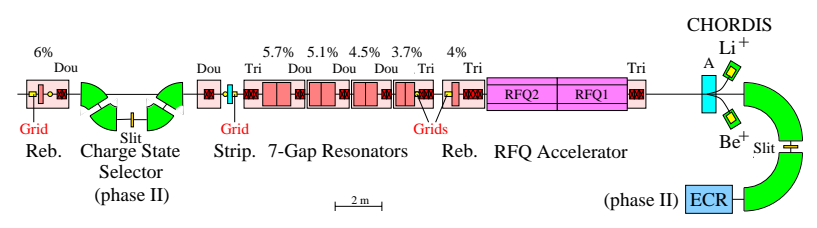

Figure 1: Schematic layout of the Heidelberg High Current Injector.

\section{INTRODUCTION}

RFQ resonators are well established structures for the acceleration of intense slow ion beams [3]. The resonance structure of a 4-rod-RFQ (see fig. 2) consists of four rods as electrodes which produce an electrical rf quadrupole field [4]. A characteristic modulation of the electrodes creates axial field components. So the resonator is able to accelerate, to bunch and to focus an ion beam with the same rf field. The main parameters of the electrodes are the aperture $\mathrm{a}$, the modulation $\mathrm{m}$ and the length of the cells $l_{c}$, which increases with the particle velocity.

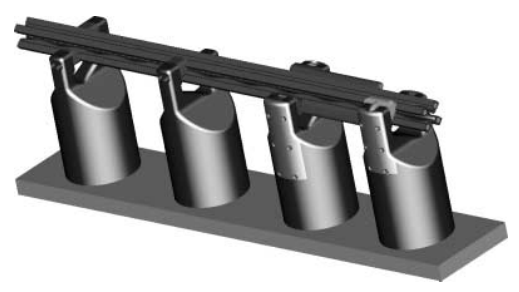

Figure 2: The resonance structure of the Heidelberg RFQ

\begin{tabular}{|l|c|c|}
\hline parameter & RFQ2 & RFQ1 \\
\hline $\mathrm{f}[\mathrm{MHz}]$ & 108.48 & 108.48 \\
Tank diameter $[\mathrm{cm}]$ & 32 & 32 \\
Tank length $[\mathrm{cm}]$ & 300 & 300 \\
$\mathrm{E}_{\text {in }}[\mathrm{keV} / \mathrm{u}]$ & 4 & 248 \\
$\mathrm{E}_{\text {out }}[\mathrm{keV} / \mathrm{u}]$ & 248 & 478 \\
$\mathrm{U}_{\text {rod }}[\mathrm{kV}]$ & 60 & 60 \\
$\mathrm{RF}$ power $[\mathrm{kW}]$ & 88 & 81 \\
Duty factor & $25 \%$ & $25 \%$ \\
q/A & $1: 9$ & $1: 9$ \\
\hline
\end{tabular}

Table 1: Parameter of the two RFQ resonators.

\section{STATIC SIMULATIONS}

With the original electrode design of the two RFQ resonators we assumed an electrode voltage of $71 \mathrm{kV}$ at $80 \mathrm{~kW}$ rf power in order to accelerate ions with a charge to mass ratio $\mathrm{q} / \mathrm{A} \geq 1 / 9$ as required for ${ }^{9} \mathrm{Be} e^{+}$. But because of the measured shunt impedance of $101 \mathrm{k} \Omega \mathrm{m}$ it was impossible to reach this design voltage. Therefore the electrodes have been redesigned for a lower voltage of $60 \mathrm{kV}$ by means of an optimization algorithm [5]. In order to achieve this voltage, we had to increase the shunt impedance about $20 \%$ by reducing the capacitance of the electrodes about $10 \%$. We

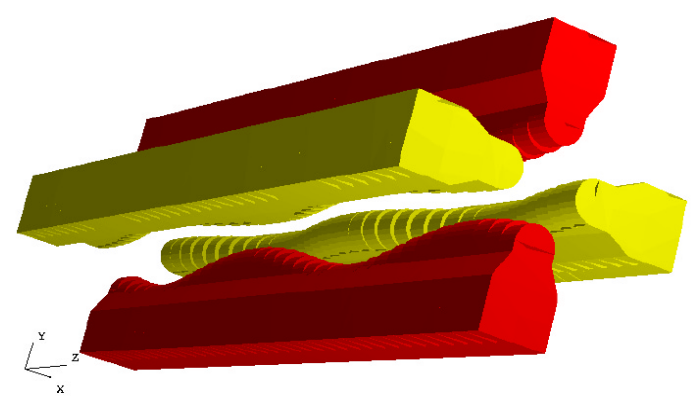

Figure 3: Input geometry of the modulated electrodes.

checked the reduction of the capacitance with three dimensional electrostatic MAFIA calculations. We calculated the capacitance per length of every cell along the electrodes.

Fig. 3 shows the three dimensional geometry with realistic values of aperture and modulation inside the mesh. Fig. 4 shows the calculated capacitance per length for the old as well as for the new electrodes. The investigation 


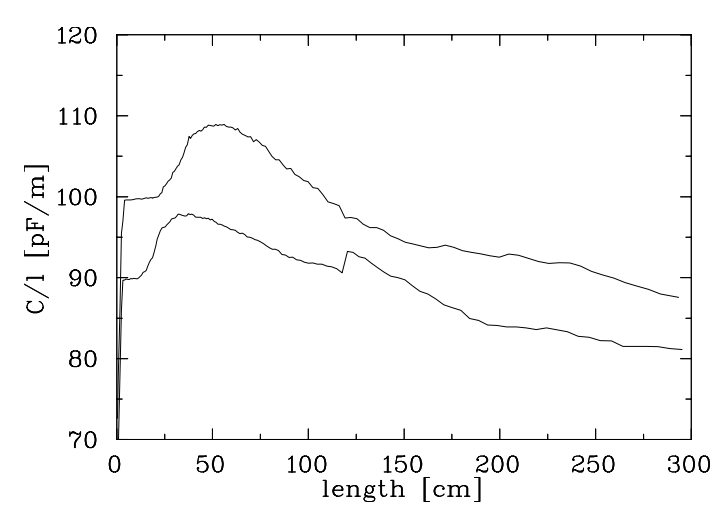

Figure 4: The calculated capacitance per length along the modulated electrodes (upper line old and lower line new electrodes).

shows that the total capacitance decreased from $284 \mathrm{pF}$ to $261 \mathrm{pF}$, which is a decrease of $8 \%$. After mounting of the new electrodes we measured the shunt impedance with a beam test. The shunt impedance increased from 101 to $121 \mathrm{k} \Omega \mathrm{m}$ for the first resonator. The rod tip of the new electrodes was milled with a $20-25 \%$ smaller radius. The smaller radius increases multipole components, but the effect on the quadrupole field has the same order of magnitude as the effect caused by a $\pm 0.2 \mathrm{~mm}$ misalignment of the rods and is tolerable [6]. We have calculated the axial electric field along the z-axis with the formula [7]

$$
E_{z}=\frac{\pi U\left(m^{2}-1\right) I_{0}(k r) \sin k z}{l_{c}\left(m^{2} I_{0}(k a)+I_{0}(m k a)\right)} .
$$

$U$ is the inter rod voltage, $a$ the aperture, $m$ the modu-

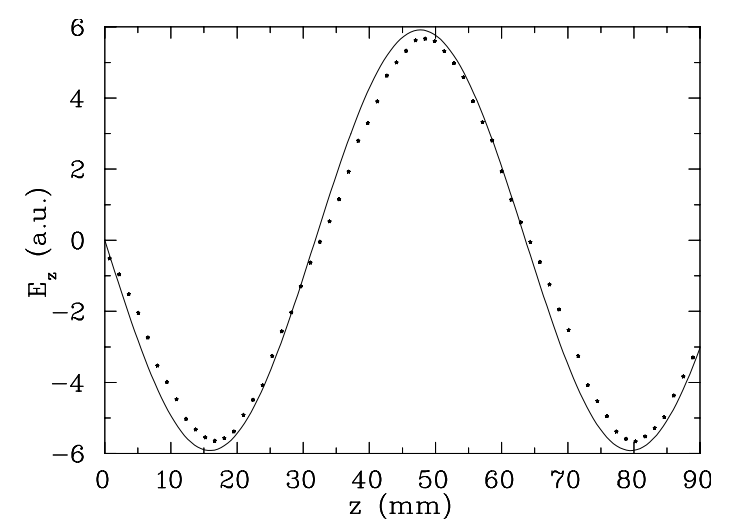

Figure 5: Calculated electric field $E_{z}$ of a typical cell with modulation. Solid line=formula with hyperbolic rod tip, asterisks=MAFIA with spherical rod tip.

lation, $k=\pi / l_{c}$, and $I_{0}$ the modified Bessel function of zero order. This formula is valid for ideal quadrupole poles i.e. hyperbolic poles. The small deviation of about $5 \%$ between the field with ideal quadrupoles and electrodes with a spherical shape allows us to use spherical electrodes to simplify the fabrication process. The increasing multipole components result in a lower strength of the axial electric field (see fig. 5).

\section{DYNAMIC SIMULATIONS}

The electrodynamic calculations of the RFQ-Resonators have been done with the Eigenmodesolver of the MAFIA version 3.23. Basically, the eigen frequency of a 4-rod RFQ is determined by one single basic cell, e.g. the length and distance of the stems and the 4 rods forming together a LCcircuit. Therefore the eigenfrequency, Q-value and shunt impedance of the quadrupole mode should be independent of the number of stems respectively the number of basic cells.

Table 2 shows the calculated and measured Q-value and

\begin{tabular}{|l|c|c|}
\hline & MAFIA & measurement \\
\hline \multicolumn{3}{|c|}{ RFQ1 } \\
\hline $\mathrm{Q}_{0}$ & 7341 & 4450 \\
\hline $\mathrm{Z}[\mathrm{k} \Omega \mathrm{m}]$ & 234.5 & 121 \\
\hline \multicolumn{3}{|c|}{ RFQ2 } \\
\hline $\mathrm{Q}_{0}$ & 7942 & 4550 \\
\hline $\mathrm{Z}[\mathrm{k} \Omega \mathrm{m}]$ & 257.5 & 131 \\
\hline
\end{tabular}

Table 2: Calculated and measured Q-value and shunt impedance of both resonators.

the shunt impedance of both Heidelberg RFQ-resonators. The calculated Q-values and shunt impedances of complicated resonators deviate typically by a factor of 2 in comparison with the measured ones [8]. But there is very good agreement of the relative values between simulation and measurement.

To investigate the voltage distribution along the accelera-

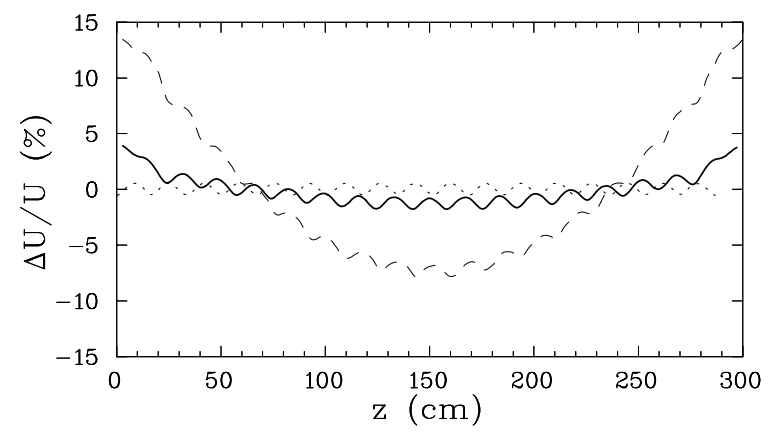

Figure 6: Calculated relative voltage distribution of a 17cell 4-rod-structure with unmodulated electrodes. Dotted line: constant distance of the stems and any edge effects. Broken line: constant distance of the stems and real length of the rods. Solid line: compensation of edge effects by reducing the length of the first and last basic cell.

tion axis, however, it is necessary to simulate the complete structure (see fig. 7). Fig. 6 shows the calculated voltage distribution between the rods along the $\mathrm{z}$ axis. The dotted line is the voltage distribution of a RFQ with constant 


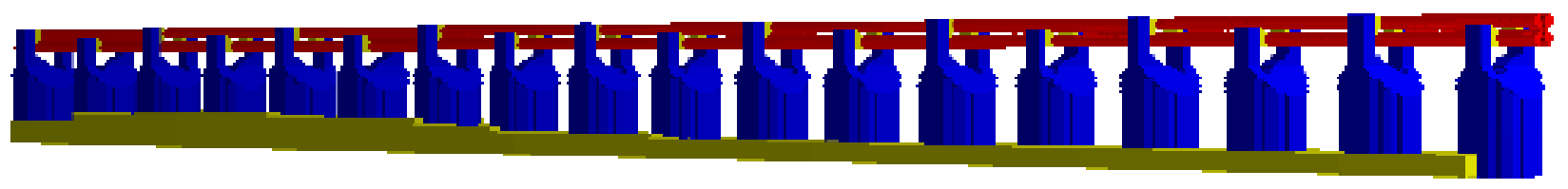

Figure 7: Input geometry of the complete RFQ 1 with variable tuning plates.

distance of the stems and without edge effects (the $x-y$ boundary planes are located in the centre of the first and last stems). An average variation of the voltage of about $0.4 \%$ reflects the characteristic of the voltage distribution of the $\lambda / 2$-resonator (maximum voltage in the middle of a stem). The broken line represents the distribution of a structure with uniform distances of the stems including the real length of the electrodes, e.g. the electrodes project out of the first and last stem. The end pieces represents an additional capacitance to ground, resulting in an unflat voltage distribution. This can be compensated by a distance reduction of the first and last stem which results in a lower inductance of the first and last basic cell. In case of the Heidelberg RFQ we have reduced the length of the edge cells from $169 \mathrm{~mm}$ to $148 \mathrm{~mm}$ (solid line).

Due to the increasing modulation along the rods and the

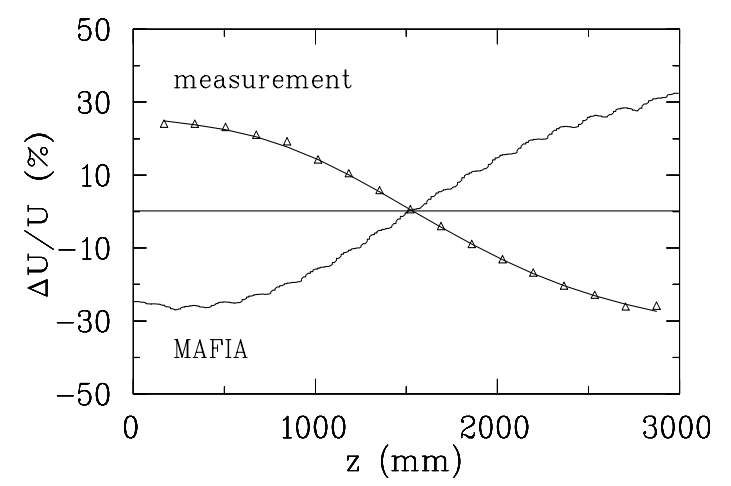

Figure 8: The relative voltage distribution along the rods was measured without tuning plates. Due to the increasing capacity the voltage distribution is unflat. The simulation has been performed with unmodulated electrodes but with tuning plates whose lead to a flat distribution. The result is an inverse voltage distribution in comparison to the measurement.

different aperture of the first RFQ the voltage distribution between the electrodes is unflat. The measured value of $\Delta U / U$ is about $18 \%$ (see Fig. 8). The eigenfrequency has been tuned with copper plates between the RFQ-stems to 108.48 MHz. The different heights of the plates influence the voltage distribution, which could be adjusted within $1 \%$ accuracy along the electrodes.

An accurate MAFIA calculation for modulated electrodes in order to determine the required tuning plates would be very time consuming. However, it is easy to calculate for unmodulated rods a distribution for different tuning plate configurations. Thus, by generating the inverse voltage distribution (see plot 'MAFIA' in Fig. 8) one can determine the tuning plate geometry necessary to compensate the experimentally measured distribution.

\begin{tabular}{|l|c|c|}
\hline structure & \multicolumn{2}{|c|}{ losses [\%] } \\
components & measurement & MAFIA \\
\hline horizontal electrodes & 30.3 & 30.5 \\
incl. 3 stems & & \\
\hline vertical electrodes & 30.3 & 30.5 \\
incl. 3 stems & & \\
\hline stems 1-6 & 15.4 & 16.9 \\
stems 7-12 & 18.8 & 16.9 \\
tank & 4.1 & 5.2 \\
\hline
\end{tabular}

Table 3: Measured and calculated power losses of different water cooled structure components of RFQ 1.

\section{CONCLUTIONS}

Extensive MAFIA calculations have been performed in order to determine important resonator parameter of the two Heidelberg RFQ accelerators. The accuracy of the absolute value of the eigen frequency was in the $2-3 \%$ range. The quality factor and shunt impedance have been calculated always a factor 2 too high. Nevertheless relative values of $\mathrm{Q}$ and $\mathrm{Z}$ have been reproduced very well.

\section{REFERENCES}

[1] R.v. Hahn et al., Nucl. Inst. and Meth., A328(1993)270-274

[2] http://www.cst.de

[3] I.M. Kapchinsky, V.A. Tepliakov, Prib. Tekh. Eksp. 2, 19-22 (1970)

[4] A. Schempp, Habilitationsschrift, Universität Frankfurt, Germany, 1990

[5] M. Madert et al., Proceedings of the LINAC conference 1996, Geneva

[6] S. Auch, MPI H-V19-1996, Master Thesis, Universität Heidelberg, 1996

[7] C.-M. Kleffner, MPI H-V24-1994, PhD Thesis, Universität Heidelberg, 1994

[8] H. Podlech, MPI H-V20-2000, PhD Thesis, Universität Heidelberg, 1999 